\section{ScienceDirect}

Materials Today: Proceedings 00 (2016) 000-000 materialstoday:
PROCEEDINGS

www.materialstoday.com/proceedings

ICMRA 2016

\title{
Printed graphene films with positive temperature coefficient of resistivity
}

\author{
Indrani Banerjee ${ }^{1,2 *}$, Tsegie Faris ${ }^{2,3}$, Zlatka Stoeva ${ }^{3}$, Ashwani K. Sharma ${ }^{4}$ and Asim K Ray ${ }^{2}$ \\ ${ }^{1}$ The Department of Physics, Birla Institute of Technology, Mesra, Ranchi-835215, INDIA \\ ${ }^{2}$ Institute of Materials and Manufacturing, Brunel University London, Uxbridge, Middlesex UB8 3PH, UK \\ ${ }^{3}$ DZP Technologies Limited, Future Business Centre, Kings Hedges Road, \\ Cambridge CB4 2HY, UK \\ ${ }^{4}$ United States Air ForceResearch Laboratory, Space Vehicles Directorate, SE Kirtland AFB, NM 87117 USA.
}

\begin{abstract}
$0.5 \mu \mathrm{m}$ thick graphene films printed on plastic substrates exhibit metallic type conduction with a positive value of $1.5 \times 10^{-3} K^{-1}$ for the temperature coefficient of resistance over the temperature range from $260 \mathrm{~K}$ to $330 \mathrm{~K}$. The crystallites of approximately $6.5 \mathrm{~nm}$ in their average size in the film are found from the X-Ray diffraction pattern to be oriented preferentially along [002] plane.
\end{abstract}

(C) 2016 Elsevier Ltd. All rights reserved.

Selection and Peer-review under responsibility of International conference on materials research and applications-2016.

Keywords: Graphene ink, resistivity, positive temperature coefficient

\section{Introduction}

Graphene is becoming increasingly popular as a result of its wide range of possible applications such as field effect transistors, sensors, transparent conductive films and supercapacitors [1]. This flexible material has very high electrical conductivity even at room temperature because of its zero bandgap where both electron and hole are involved in charged carriers [2]; the presence of pi $(\pi)$ electrons in the above and below the graphene flat sheet and their hexagonal lattice structures make the charge carriers mobility to be high[3] [4]. Graphene exists in different structures and morphologies and their conductivities are different to each other. Complete exfoliation of the

\footnotetext{
* Corresponding author. Tel.: +91 9470564719; fax+9106512275401 .

E-mail address: indranibanerjee@bitmesra.ac.in
} 
graphitic structure with 2D graphene sheets leads to increase of the electrical conductivity of graphene [5].

The carrier density can be improved by doping however doping may affect the stability of graphene [6]. Techniques such as processing at very high temperature and growing a large grains size are also used to improve conductivity; however these techniques are not always suited to practical applications [6]. Mechanical exfoliation and chemical vapour deposition (CVD) are commonly used to synthesize high quality graphene films with reduced number of defects by annealing [7]; however these methods are not suitable for deposition over large areas.

Printing can be used as an alternative technique to make conductive graphene films. Some of the advantages of graphene printing are the low processing temperature at ambient conditions without using vacuum or inert atmosphere. The other benefit of the graphene printing is that films can be obtained over large areas on flexible substrates, at a reduced cost [8]. The main interest of this paper was to examine conductivity of commercially available graphene ink. The samples were characterized XRD spectroscopy together with resitance measurements which were taken inside the cryostat under vacuum at deferent temperatures.

\section{Experimental procedure}

The graphene ink used in this work was supplied by DZP technologies Ltd, product number G087. The ink was formulated with few-layer graphene particles of lateral dimensions $<40$ microns, which were obtained by liquid phase exfoliation. The graphene particles were dispersed in acrylic polymer system to obtain the ink suitable for deposition on plastic substrates. Silver printed interdigitated electrodes were fabricated on plastic substrates prior to graphene ink deposition. The samples were characterized by means of X-Ray diffraction using Bruker D8 advance scanning instrument. The $\mathrm{Cu} \mathrm{K}$ radiation of wavelength $0.15406 \mathrm{~nm}$ was used with scattering angle varied between $10^{0}-90^{\circ}$ at scanning rate of 0.02 per sec for crystallographic analysis of the samples. The electrical properties of the sample were determined from the electrometer with the sample loaded inside the cryostat maintained under vacuum and at different temperatures from $-5^{\circ} \mathrm{C}$ to $60^{\circ} \mathrm{C}$.

\section{Results and discussions}

The crystallographic analysis of the graphene ink was performed by XRD. Figure 1 shows the XRD spectrum of the sample with major reflection at $2 \theta=26.19^{0}$ corresponding to the reflection from [002] plane of graphite [9].

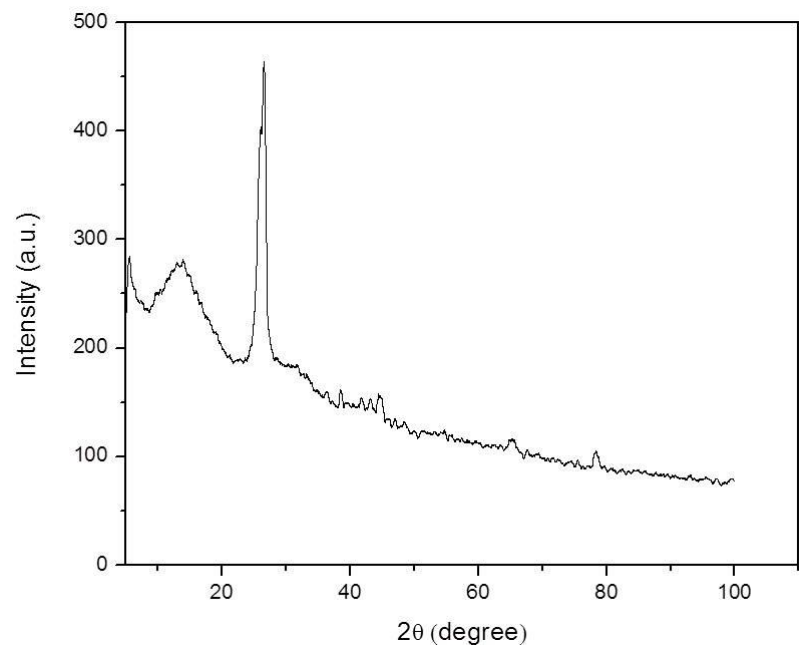

Figure1: XRD spectrum of the sample

The interlayer " $\mathrm{d}$ " spacing of the sample was estimated to be $\sim 0.34 \mathrm{~nm}$ which is close to the conventional graphitic plane. The crystallite size has been estimated from the Debye Scherrer formula to be $\sim 6.46 \mathrm{~nm}$. The sample showed an additional weak peak at $2 \theta=13^{0}$ corresponding the reflection from [010] plane of graphene oxide. This indicates the presence of residual oxygenated functional components in the crystalline matrix of graphene ink. 


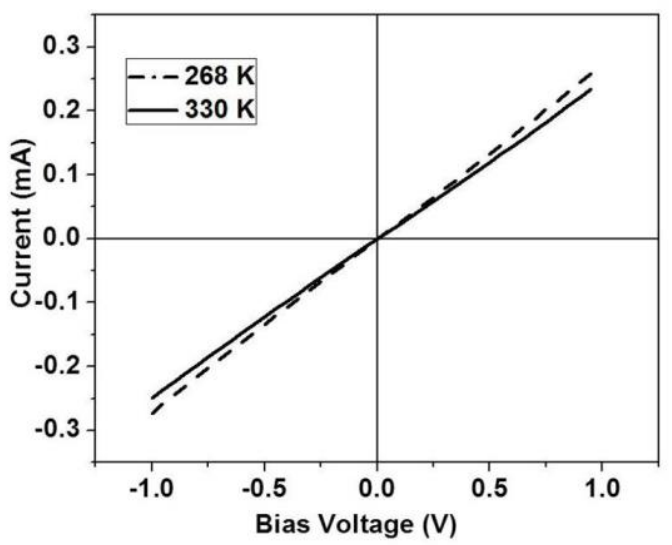

Figure 2: $\mathrm{I}(V)$ characteristics for $\mathrm{I}(V)$ characteristics of graphene ink sample for $\mathrm{T}=268 \mathrm{~K}$ (broken line) and $\mathrm{T}=300 \mathrm{~K}$ (solid line).

Figure 2 shows the current I versus bias voltage $\mathrm{V}$ plots for the sample at $\mathrm{T}=268 \mathrm{~K}$ and $\mathrm{T}=330 \mathrm{~K}$. These $\mathrm{I}(V)$ characteristics are linear within the bias voltage regime, implying the Ohmic nature of conduction. Further measurements are repeated for four different temperatures between these two temperatures. Values of dark Ohmic resistance $R_{D}$ at were determined different temperatures from $T=268 \mathrm{~K}$ to $T=330 \mathrm{~K}$ from the slope of individual $\mathrm{I}(V)$ plot and the dependence of $\mathrm{R}_{\mathrm{D}}$ on temperature $\mathrm{T}$ is shown in Figure 3. The behaviour can be described by a simple well-known relation in the form:

$$
R_{D}=R_{D 0}[1+\propto T]
$$

where $\mathrm{R}_{D 0}$ and $\alpha$ denote the resistance at $0 \mathrm{~K}$ and temperature coefficient of resistivity the GI sample. The ratio of the slope to the intercept at $\mathrm{T}=0 \mathrm{~K}$ gives $1.5 \times 10^{-3} / \mathrm{C}$ for temperature coefficient $\alpha$.

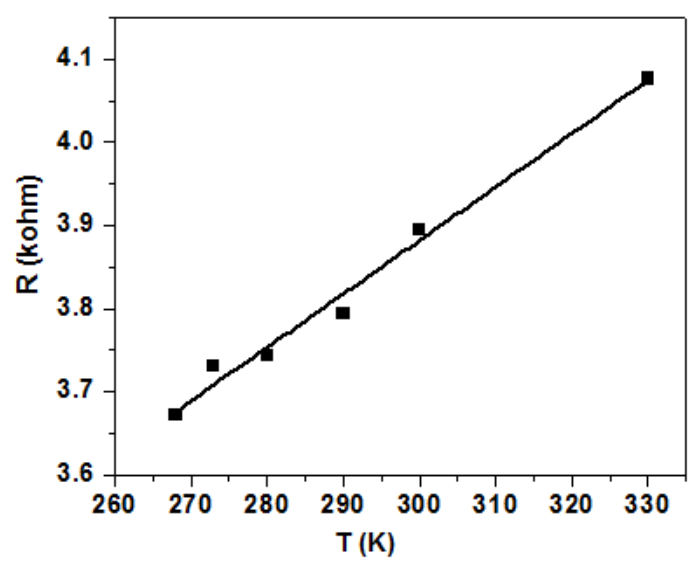

Figure 3: Dependence of Resistivity (R) on Temperature (T) 
The positive temperature coefficient is a signature for conducting materials. This value is of the same order of magnitude as $3.9 \times 10^{-3} \mathrm{C}^{-1}$ and $3.4 \times 10^{-3} \mathrm{C}^{-1}$ for highly conducting metals like copper and silver.

\section{Conclusions}

A low cost easy fabrication printed graphene samples have been developed and tested for it optoelectric properties. For flexible electronics the temperature coefficient of resistance plays significant role in applications. Small temperature coefficient is expected to be as low as possible for shielding from electromagnetic interference applications to ensure stable electrical performance. Conducting polymer composites loaded with graphene powder is often used for such applications.

\section{Acknowledgements}

Dr. Indrani Banerjee is grateful to Commonwealth Association, UK for funding the present research work under the fellowship placement scheme (grant reference INCF-2014-66). The studentship of Ms Faris is partially sponsored by the Air Force Office of Scientific Research, Air Force Material Command, USAF, under Grant No. FA8655-08$1-3056$.

\section{References}

[1]M. Yang, Y. Hou, N. A. Kotov, “Graphene-based multilayers: Critical evaluation of materials assembly techniques,” Nano Today, Vol.7,pp. 430-447, Oct. 2012

[2] I. N. Kholmanov, C. Magnuson, A. E. Aliev, H Li, B. Zhang, J.W.Suk, L. L. Zhang, E. Peng, S. H. Mousavi,A. B. Khanikaev, R. Piner, G. Svets, and R. S. Ruoff, "Improved Electrical Conductivity of Graphene Films Integrated with Metal Nanowires," Nano Letters, vol. 12, pp. 56795683 Oct. 2012

[3] J. M. Poumirol, W. Escoffier, A .Kumar, M .Goiran, B .Raquet and J .M .Broto,"Electron-hole coexistence in disordered graphene probed by high-field magneto-transport” New J. Phys. vol. 12,Aug. 2010

[4] Y. L. Shen, P. Zhou, L. H. Wang, Q. Q. Sun, Q. Q. Tao, P. F. Wang, S. J. Ding, D. W. Zhang," The Annealing Effect of Chemical Vapor Deposited Graphene," IEEE ,Oct. 2013

[4b] A. Pirkle, J. Chan, A. Venugopal, D. Hinojos, C. W. Magnuson, S. McDonnell, L. Colombo, E. M. Vogel, R. S. Ruoffand R. M. Wallace, "The effect of chemical residues on the physical and electrical properties of chemical vapor deposited graphene transferred to SiO2," Appl. Phys. Lett.,vol. 99, pp.122108, June. 2011

[5] E. Pop , V. Varshney, and A. K. Roy “Thermal properties of graphene: Fundamentals and applications," MRS Bull ,Vol. 37, pp 12731281, dec. 2012

[6] Y. Raj and S.N.Lakshminarasimhan "Advance in composite materials- graphene and its applications in modern composite world" IJCRR, vol.2,Oct 2014

[7] F. Bonaccorso, L.Lolombo, Gu. Yu, M. Stoller, V. Tozzini, AC . Ferrari, RS. Ruoff and V.pellegrini,“Graphene, related two-dimensional crystals, and hybrid systems for energy conversion and storage" Science 2 vol.347, no. 6217 , DOI: 10.1126/science.1246501,Jan 2015

[8] T. Kobayashi, M. Bando, N. Kimura, K. Shimizu, K. Kadono, N. Umezu, K.Miyahara, S. Hayazaki, S. Nagai, Y. Mizuguchi, Y. Murakami, and D. Hobara,"Production of a 100-m-long high-quality graphene transparent conductive film by roll-to-roll chemical vapor deposition and transfer process," Appl. Phys. Lett., Vol.102, pp.023112 -4, Jan 2013

[9] S.Keun-Young, H.Jin-Yong and J. Jyongsik "Flexible and transparent graphene films as acoustic actuator electrodes using inkjet printing “ Chem. Commun. ,Vol. 47, pp.8527-8529,DOI: 10.1039/c1cc12913a ,June 2011. 